\title{
Comparison of Model Fit Indices Used in Structural Equation Modeling Under Multivariate Normality
}

Sengul Cangur

Duzce University, Duzce, Turkey, sengulcangur@duzce.edu.tr

Ilker Ercan

Uludag University, Bursa, Turkey, ercan@uludag.edu.tr

Follow this and additional works at: http://digitalcommons.wayne.edu/jmasm

Part of the Applied Statistics Commons, Social and Behavioral Sciences Commons, and the Statistical Theory Commons

\section{Recommended Citation}

Cangur, Sengul and Ercan, Ilker (2015) "Comparison of Model Fit Indices Used in Structural Equation Modeling Under Multivariate Normality," Journal of Modern Applied Statistical Methods: Vol. 14 : Iss. 1, Article 14.

DOI: $10.22237 /$ jmasm/1430453580

Available at: http://digitalcommons.wayne.edu/jmasm/vol14/iss1/14

This Regular Article is brought to you for free and open access by the Open Access Journals at DigitalCommons@WayneState. It has been accepted for inclusion in Journal of Modern Applied Statistical Methods by an authorized editor of DigitalCommons@WayneState. 


\title{
Comparison of Model Fit Indices Used in Structural Equation Modeling Under Multivariate Normality
}

\author{
Sengul Cangur \\ Duzce University \\ Duzce, Turkey
}

\author{
Ilker Ercan \\ Uludag University \\ Bursa, Turkey
}

\begin{abstract}
The purpose of this study is to investigate the impact of estimation techniques and sample sizes on model fit indices in structural equation models constructed according to the number of exogenous latent variables under multivariate normality. The performances of fit indices are compared by considering effects of related factors. The Ratio Chi-square Test Statistic to Degree of Freedom, Root Mean Square Error of Approximation, and Comparative Fit Index are the least affected indices by estimation technique and sample size under multivariate normality, especially with large sample size.
\end{abstract}

Keywords: Structural equation modeling, multivariate normality

\section{Introduction}

Modeling methods are employed for studying the phenomena than require the utilization of complex variable set. Structural Equation Modeling (SEM) is preferred when studying the causal relations and the latent constructs among the variables is in question. The reason is it can be used to analyze complex theoretical models and its practicability.

The objective of SEM is to explain the system of correlative dependent relations between one or more manifest variables and latent constructs simultaneously. It serves to determine how the theoretical model that denotes relevant systems is supported by sample data, i.e., estimation of relations between the main constructs. Because there is no single criterion for the theoretical model fit evaluation obtained as a result of SEM, a wide array of fit indices was developed (Schermelleh-Engel and Moosbrugger, 2003; Ding et al., 1995; Sugawara and MacCallum, 1993). Studies conducted through SEM were

Dr. Cangur is an Assistant Professor in the Department of Biostatistics and Medical Informatics. Email at sengulcangur@duzce.edu.tr. Dr. Ercan is a Professor in the Department of Biostatistics. Email at ercan@uludag.edu.tr. 


\section{CANGUR \& ERCAN}

undertaken by using empirical and non-empirical data so as to develop and confirm theory (Bentler and Dudgeon, 1996; Wang et al., 1996; Bentler, 1994).

Simulation studies were conducted to test the robustness of SEM, because the assumptions required usually cannot be verified in practice. Because these studies were conducted in order to verify hypothesis, a known theoretical model was taken as a reference and the behaviors of the most commonly used techniques in specific conditions were observed. The parameter estimations obtained through the estimation techniques based on various distributional conditions and sample size, standard errors and the bias of model fit indices were researched in the studies conducted.

Studies were conducted for recommending and improving the parameter estimation techniques used in SEM and selecting the conditions in which these are to be used (Boomsma and Hoogland, 2001; Wang et al., 1996; Chou and Bentler, 1995; Bentler, 1994). Other studies were conducted by employing various empirical designs so as to examine the effects of factors such as estimation techniques, sample sizes, distributional conditions, number of latent variables, number of manifest variables, the misspecification degree of the model, factor loads, factor correlations, improper solutions, convergence errors on model fit indices make contribution to the SEM literature (e.g., Herzog \& Boomsma, 2009; Fan \& Sivo, 2007; Sivo et al., 2006; Lei \& Lomax, 2005; Marsh et al., 2004; Boomsma and Hoogland, 2001; Fan et al., 1999; Hu \& Bentler, 1998, 1999; Wang et al., 1996; Chou and Bentler, 1995; Ding et al., 1995; Marsh \& Balla, 1994; Sugawara and MacCallum, 1993; Gerbing \& Anderson, 1992).

Hence, a wide array of simulation studies were conducted on model fit indices through various estimation techniques. Unlike these studies, in the current study the inclusion of a higher number of estimation techniques was used. Furthermore, the differentiation of the model structure was agreed to be studied as exogenous factor rather than an effect so as to reach a mutual interpretation. The effects of estimation technique and sample size factors on model fit indices were examined in circumstances in which the multivariate normality assumption was ensured and in the models which were established by taking exogenous (independent) latent variables into consideration in the research. The model fit indices were compared to recommend appropriate model fit indices in line with the effects of these factors. 


\section{COMPARISON OF MODEL FIT INDICES}

\section{Methodology}

\section{Maximum likelihood estimation technique}

Maximum likelihood estimation (MLE) technique is one of the normal theory estimation techniques that is able to provide model parameter estimations simultaneously (Kline, 2011; Chou and Bentler, 1995). Assume a $\left\{\mathbf{x}_{1}, \mathbf{x}_{2}, \ldots, \mathbf{x}_{n}\right\}$ random sample is derived from multivariate normal distribution $\mathrm{N}\left(\boldsymbol{\mu}_{0}, \boldsymbol{\Sigma}_{0}\right)$. In order to achieve $\boldsymbol{\Sigma}_{0}=\boldsymbol{\Sigma}\left(\boldsymbol{\theta}_{0}\right)$, assumed there is population (true) matrix function with $\boldsymbol{\Sigma}_{0}, q \times 1$ size and $\boldsymbol{\theta}_{0}$ unknown parameter. In this case, MLE function can be defined as in equation (1).

$$
\mathrm{F}_{\mathrm{MLE}}(\boldsymbol{\theta})=\log |\boldsymbol{\Sigma}(\boldsymbol{\theta})|+\operatorname{tr}\left(\mathbf{S} \boldsymbol{\Sigma}(\boldsymbol{\theta})^{-1}\right)-\log |\mathbf{S}|-p
$$

S denotes sample covariance matrix while $\boldsymbol{\Sigma}\left(\boldsymbol{\theta}_{0}\right)$ indicates the covariance matrix of the hypothesized model, $t r$ denotes the trace of matrix and $p$ represents the number of manifest variables (Lee, 2007).

\section{Generalized least squares technique}

The GLS technique makes multivariate normality assumption flexible compared to MLE technique, yet also features the assumptions of MLE technique. GLS function can be given as follows.

$$
\mathrm{F}_{\mathrm{GLS}}(\boldsymbol{\theta})=2^{-1} \operatorname{tr}\{(\mathbf{S}-\boldsymbol{\Sigma}) \mathbf{V}\}^{2}
$$

The population and sample covariance matrices are indicated with $\boldsymbol{\Sigma}$ and $\mathbf{S}$ respectively. The $\mathbf{V}$ matrix can be a constant positive definite matrix or a stochastic matrix which converges to $\Sigma_{0}^{-1}$. The GLS function reduces to the least squares function when $\mathbf{V}$ equals to identity matrix (I) (Lee, 2007).

\section{Asymptotically distribution-free technique}

The Asymptotically Distribution-Free (ADF) technique does not require multivariate normality assumption and is based on the calculation of $\mathbf{W}$ weighted matrix and GLS estimation. Accordingly, assume $\mathbf{x}_{1}, \mathbf{x}_{2}, \ldots, \mathbf{x}_{n}$ are the independent identically distributed observations of a sample with mean vector $\mu$, covariance matrix $\boldsymbol{\Sigma}_{0}=\boldsymbol{\Sigma}\left(\boldsymbol{\theta}_{0}\right)$ and finite eighth-order moments that is not obliged 


\section{CANGUR \& ERCAN}

to be selected from a multivariate normal distribution. $\tilde{\boldsymbol{\theta}}_{\boldsymbol{A}}$ ADF estimator of $\boldsymbol{\theta}_{0}$ will be defined as in equation (3) as the vector which minimizes GLS function:

$$
\mathrm{F}_{\mathrm{ADF}}(\boldsymbol{\theta})=2^{-1}[\operatorname{vecs}\{\mathbf{S}-\boldsymbol{\Sigma}(\boldsymbol{\theta})\}]^{\prime} \mathbf{W}^{-1}[\operatorname{vecs}\{\mathbf{S}-\boldsymbol{\Sigma}(\boldsymbol{\theta})\}]
$$

Here vecs denotes the column vector which is obtained through derivation of lower triangle matrix components row by row. $\mathbf{W}$ is the stochastic weighted matrix with positive definite and is assumed to converge to $\Sigma^{*}$ (Lee, 2007). Many researchers emphasized the requirement to work with large sample sizes so as to ensure that $\mathrm{ADF}$ estimations have the desired asymptotical properties (i.e., Bentler \& Dudgeon, 1996).

\section{Satorra-Bentler scaled chi square test statistic}

The normal theory chi-square statistic can be adjusted for its convergence to the referenced chi-square distribution even if it is not fit for the expected chi-square distribution in circumstances where the normality assumption is violated. Satorra-Bentler scaled $\chi^{2}$ test statistic can be indicated as follows:

$$
\chi_{\mathrm{SB}}^{2}=\frac{\chi_{\mathrm{MLE}}^{2}}{\varpi}
$$

$\chi_{\text {MLE }}^{2}$ denotes the chi-square value of MLE technique. The $\varpi$ constant, also known as the scaling factor, is a function of the model-implied weighted matrix, the multivariate kurtosis index and the degree of freedom for the model (Finney and Distefano, 2006; Chou and Bentler, 1995). Provided that multivariate kurtosis is not in question $\chi_{\mathrm{MLE}}^{2}$ value is equal to $\chi_{\mathrm{SB}}^{2}$ value, and two chi-square values are obtained as different from each other only on the event of the degree of multivariate kurtosis increases (Finney and Distefano, 2006).

\section{Commonly-used model fit indices in SEM}

$\chi^{2}$ and $\chi^{2} / \boldsymbol{v}$ Ratio The $\chi^{2}$ test statistic is an absolute fit index which assumes multivariate normality and is sensitive to sample size (Gerbing and Anderson, 1992). This test statistic 


\section{COMPARISON OF MODEL FIT INDICES}

$$
\chi^{2}=-2\left\{-1 / 2(n-1)\left[\operatorname{tr}\left(\mathbf{S} \Sigma^{-1}\right)+\log |\Sigma|-\log |\mathbf{S}|-p\right]\right\}=(n-1) \mathrm{F}
$$

is distributed the central $\chi^{2}$ with degree of freedom $\{1 / 2 p(p+1)\}-t$ in large samples. Here $p$, denotes the number of observed variables and $t$ symbolizes the number of estimated independent parameters. $\mathbf{S}$ denotes unrestricted sample covariance matrix whereas $\boldsymbol{\Sigma}(\boldsymbol{\theta})$ denotes restricted covariance matrix. It is said that the larger the likelihood related to $\chi^{2}$, the closer the fit between the hypothesized model and the perfect model (Herzog and Boomsma, 2009; Hu and Bentler, 1995). This statistic is dependent on sample size. With increasing sample size and a fixed number of degree of freedom, the $\chi^{2}$ value increases. This signs to the problem that plausible models might be rejected (Schermelleh-Engel and Moosbrugger, 2003).

$\chi^{2} / v, \chi^{2}$ is an index obtained by dividing the test statistic value by the degree of freedom $(v)$. It is known as parsimony and stand-alone fit index. The development of Tucker-Lewis Index is also based on this ratio. The value of this ratio gives information on the fit between data and model. It is said that with smaller index value of $\chi^{2} / v$ ratio, the consistency will be better. SchermellehEngel and Moosbrugger (2003) stated that this ratio indicates good fit when it produces 2 or a smaller value while it indicates an acceptable value when it produces a value of 3 . Ding et al. (1995) stated that this ratio should be close to 1 or have a smaller value.

\section{Standardized Root Mean Square Residual (SRMR) Index The}

Standardized Root Mean Square Residual (SRMR) is an index of the average of standardized residuals between the observed and the hypothesized covariance matrices (Chen, 2007). This absolute fit index can be indicated as follows:

$$
S R M R=\sqrt{\frac{\sum_{i=1}^{p} \sum_{j=1}^{i}\left[\left(s_{i j}-\hat{\sigma}_{i j}\right) /\left(s_{i i} s_{j j}\right)\right]^{2}}{p(p+1) / 2}}
$$

where $s_{i j}$ indicates a component of $\mathbf{S}$ sample covariance matrix and $\hat{\sigma}_{i j}$ shows a component of $\Sigma(\hat{\theta})$ hypothesized model whereas $p$ is the number of observed variables. SRMR does not give any information about the direction of 


\section{CANGUR \& ERCAN}

discrepancies between $\mathbf{S}$ and $\boldsymbol{\Sigma}(\hat{\boldsymbol{\theta}})$ (Kline, 2011; Schermelleh-Engel and Moosbrugger, 2003).

Although SRMR indicates the acceptable fit when it produces a value smaller than 0.10, it can be interpreted as the indicator of good fit when it produces a value lower than 0.05 (Kline, 2011; Hu and Bentler, 1999; Schermelleh-Engel and Moosbrugger, 2003; Lacobucci, 2010). One of the reasons of preferring SRMR index in studies is its relative independence from sample size (Chen, 2007).

\section{Root Mean Square Error of Approximation (RMSEA) Index The}

RMSEA is an index of the difference between the observed covariance matrix per degree of freedom and the hypothesized covariance matrix which denotes the model (Chen, 2007). This absolute fit index is estimated as follows:

$$
\text { RMSEA }=\sqrt{\max \left\{\left(\frac{\mathrm{F}(\mathbf{S}, \Sigma(\hat{\boldsymbol{\theta}}))}{v}-\frac{1}{n-1}\right), 0\right\}}
$$

Here $\mathrm{F}(\mathbf{S}, \boldsymbol{\Sigma}(\hat{\boldsymbol{\theta}}))$ indicates the fit function is minimized whereas max points to the maximum value of the values given in brackets. While $l$ is the number of known parameters and $t$ is the number of independent parameters, $v=l-t$ indicates the value of the degrees of freedom and $n$ indicates the sample size (SchermellehEngel and Moosbrugger, 2003).

Observe in equation (7) that RMSEA produces a better quality of estimation when the sample size is large compared to smaller sample sizes. When the sample size is large, the term [1/(n-1)] gets closer to zero asymptotically (Rigdon, 1996).

The RMSEA also takes the model complexity into account as it reflects the degree of freedom as well. RMSEA value smaller than 0.05 , it can be said to indicate a convergence fit to the analyzed data of the model while it indicates a fit close to good when it produces a value between 0.05 and 0.08 . A RMSEA value falling between the range of $0.08-0.10$ is stated to indicate a fit which is neither good nor bad. Hu and Bentler (1999) remarked that RMSEA index smaller than 0.06 would be a criterion that will suffice. A few researchers stated that RMSEA is among the fit indexes which are affected the least by sample size (Marsh et al., 2004; Schermelleh-Engel and Moosbrugger, 2003). 


\section{COMPARISON OF MODEL FIT INDICES}

Tucker-Lewis Index (TLI) The Tucker-Lewis Index (TLI) is an incremental fit index. Non-Normed Fit Index (NNFI) which is also known as TLI was developed against the disadvantage of Normed Fit Index regarding being affected by sample size. TLI is calculated as given below (Schermelleh-Engel and Moosbrugger, 2003; Ding et al., 1995; Gerbing \& Anderson, 1992).

$$
T L I=\frac{\left(\chi_{i}^{2} / v_{i}\right)-\left(\chi_{t}^{2} / v_{t}\right)}{\left(\chi_{i}^{2} / v_{i}\right)-1}=\frac{\left(\mathrm{F}_{i} / v_{i}\right)-\left(\mathrm{F}_{t} / v_{t}\right)}{\left(\mathrm{F}_{i} / v_{i}\right)-(1 /(n-1))}
$$

Here $\chi_{i}^{2}$ belongs to the independence model whereas $\chi_{t}^{2}$ belongs to the target model. $v_{i}$ and $v_{t}$ are the number of degrees of freedom for the independence and target models respectively, in relation to the chi-square test statistics. F is the value of appropriate minimum fit function, and $n$ indicates sample size.

The bigger TLI value indicated better fit for the model. Although values larger than 0.95 are interpreted as acceptable fit, 0.97 is accepted as the cut-off value in a great deal of researches. Furthermore TLI is not required to be between 0 and 1 as it is non-normed. The key advantage of this fit index is the fact that it is not affected significantly from sample size (Schermelleh-Engel and Moosbrugger, 2003; Ding et al., 1995; Gerbing \& Anderson, 1992).

Comparative Fit Index (CFI)

The Comparative Fit Index (CFI) is an incremental fit indices. CFI is a corrected version of relative non-centrality index. The extent to which the tested model is superior to the alternative model established with manifest covariance matrix is evaluated (Chen, 2007) and the equation can be given as in (9).

$$
C F I=1-\frac{\max \left[\left(\chi_{t}^{2}-v_{t}\right), 0\right]}{\max \left[\left(\chi_{t}^{2}-v_{t}\right),\left(\chi_{i}^{2}-v_{i}\right), 0\right]}
$$

Here max indicates the maximum value of the values given in brackets. $\chi_{i}^{2}$ and $\chi_{t}^{2}$ are test statistics of the independence model and the target model respectively. $v_{i}$ and $v_{t}$ are the degrees of freedom of the independence model and the target model in relation to chi-square test statistics respectively (SchermellehEngel and Moosbrugger, 2003; Ding et al., 1995; Gerbing \& Anderson, 1992). 


\section{CANGUR \& ERCAN}

The CFI produces values between $0-1$ and high values are the indicators of good fit. When CFI value is 0.97 , it means that the fit in question is better compared to the independence model. An acceptable fit is in question provided that CFI value is larger than 0.95 (Schermelleh-Engel and Moosbrugger, 2003). This index is relatively independent from sample size and yields better performance when studies with small sample size (Chen, 2007; Hu and Bentler, 1998).

\section{Hypothesized models}

Two structural equation models (SEMs) with different structures of mean and covariance, and constructed in accordance with exogenous latent variable number were established. Model 1 is the model with two exogenous and one endogenous latent variables with each of the exogenous variable having two indicators (Figure 1). Model 2 is the other model established through the addition of one exogenous variable with two indicators to the structure given in Model 1 (Figure 2).

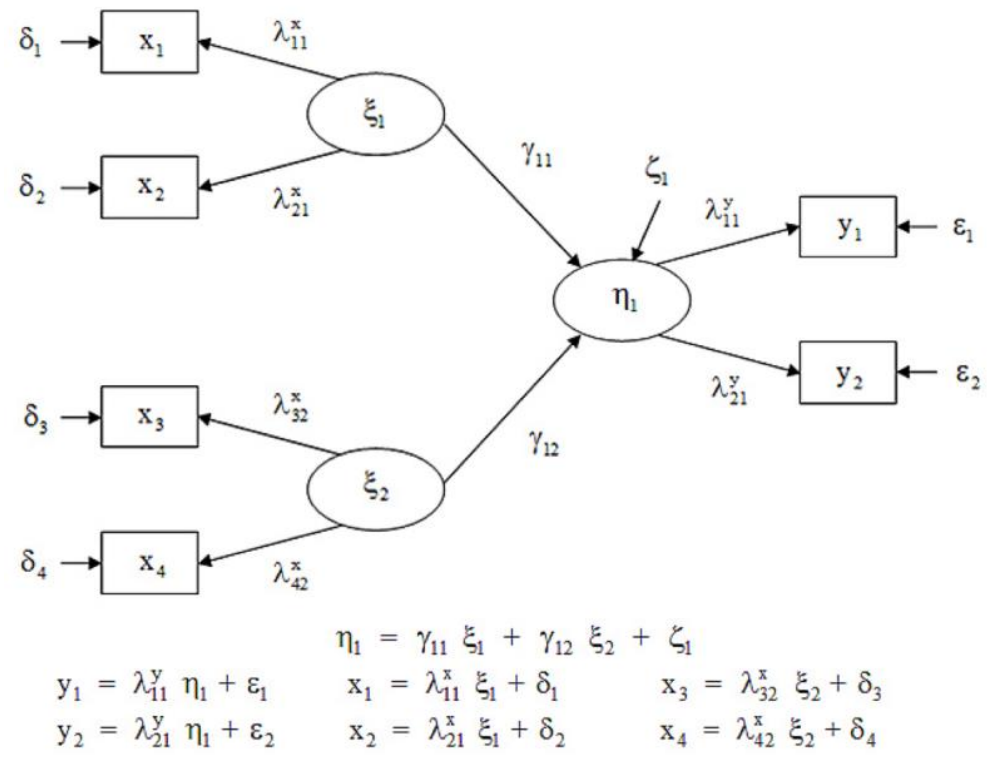

Figure 1. Structural equation model with three latent variables, with observed variables each (Model 1) 


\section{COMPARISON OF MODEL FIT INDICES}

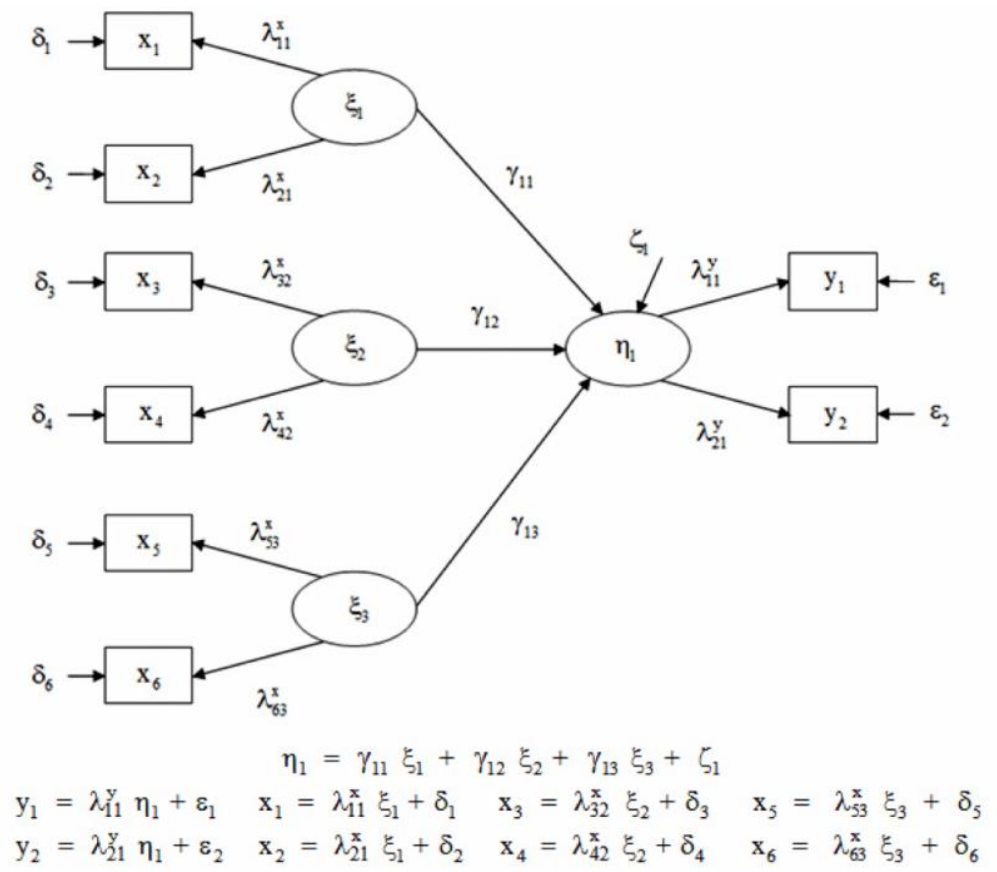

Figure 2. Structural equation model with four latent variables, with observed variables each (Model 2)

\section{Sample generation}

The mean vectors and covariance matrices which were used for generating data are given in Table 1 for identification model. Multivariate normal distribution data were generated by taking Model 1 and Model 2 into consideration for the sample sizes determined as 100, 500 and 1000 units. MLE, GLS, ADF and SB_ $\chi^{2}$ techniques were applied to the derived data. SEMs which are significant in accordance to the test statistics were included in the study $(p>0.05) \cdot \chi^{2} / v$ ratio, SRMR, RMSEA, TLI, and CFI model fit indices which were obtained from the significant SEMs were recorded. A total of 1200 significant SEMs were examined in the research. The simulation and all of the remaining statistical analyses were performed in $\mathrm{R}$ software through the utilization of MSBVAR, mvShapiroTest, QRMlib and lavaan packages. 


\section{CANGUR \& ERCAN}

Table 1. Covariance matrices and Mean vectors of Model 1 and Model 2

\begin{tabular}{|c|c|c|c|c|c|c|c|c|}
\hline Model 1 & & $\mathbf{y}_{1}$ & $\mathrm{y}_{2}$ & $\mathbf{x}_{1}$ & & & $\mathbf{X}_{3}$ & $\mathbf{X}_{4}$ \\
\hline $\mathbf{y}_{1}$ & & 1.50 & & & & & & \\
\hline $\mathbf{y}_{2}$ & & 1.18 & 1.50 & & & & & \\
\hline $\mathbf{x}_{1}$ & & 0.95 & 0.90 & 1.50 & & & & \\
\hline $\mathbf{X}_{2}$ & & 0.95 & 0.90 & 1.20 & & & & \\
\hline $\mathbf{X}_{3}$ & & 0.95 & 0.90 & 0.50 & & & 1.50 & \\
\hline $\mathbf{X}_{4}$ & & 0.95 & 0.90 & 0.50 & & & 1.30 & 1.50 \\
\hline$\mu_{1}=$ & & $(100$ & 100 & 100 & & & 100 & 100) \\
\hline Model 2 & $\mathbf{y}_{1}$ & y2 & $\mathbf{x}_{1}$ & $\mathbf{X}_{2}$ & $\mathbf{X}_{3}$ & $\mathbf{X} 4$ & $\mathbf{X}_{5}$ & $\mathrm{X}_{6}$ \\
\hline $\mathbf{y}_{1}$ & 1.50 & & & & & & & \\
\hline $\mathrm{y}_{2}$ & 1.18 & 1.50 & & & & & & \\
\hline $\mathbf{x}_{1}$ & 0.95 & 0.90 & 1.50 & & & & & \\
\hline $\mathbf{X}_{2}$ & 0.95 & 0.90 & 1.20 & 1.50 & & & & \\
\hline $\mathbf{x}_{3}$ & 0.95 & 0.90 & 0.50 & 0.50 & 1.50 & & & \\
\hline $\mathbf{X}_{4}$ & 0.95 & 0.90 & 0.50 & 0.50 & 1.30 & 1.50 & & \\
\hline$X_{5}$ & 0.95 & 0.90 & 0.50 & 0.50 & 0.50 & 0.50 & 1.50 & \\
\hline $\mathrm{X}_{6}$ & 0.95 & 0.90 & 0.50 & 0.50 & 0.50 & 0.50 & 1.25 & 1.50 \\
\hline$\mu_{2}=$ & $(100$ & 100 & 100 & 100 & 100 & 100 & 100 & $100)$ \\
\hline
\end{tabular}

$\mu_{1}$ : Mean vector of Model 1; $\mu_{2}$ : Mean vector of Model 2

\section{Study design}

The study was designed as $4 \times 3$ so as to examine the effects of 4 different estimation techniques (MLE, GLS, ADF and $\mathrm{SB}_{-} \chi^{2}$ ) and 3 different sample sizes $(100,500$ and 1000) under multivariate normal distribution condition by taking both structural models into consideration.

A rank transform was applied to each index, and then Factorial Analysis of Variance (Factorial ANOVA) was conducted so as to find out the effects of estimation technique and sample size factors on $\chi^{2} / v$ ratio, SRMR, RMSEA, TLI and CFI model fit indices based on the models established. Tukey's Honestly Significant Difference (Tukey's HSD) was used for the pairwise comparisons of the factors in which statistically significant differences were found.

\section{Results}

Out of the simulation results obtained by applying SEM estimation techniques to Model 1 and Model 2 under multivariate normality condition, 3.17\%, 8.60\% and $7.6 \%$ comprise of the convergence error of model, improper solutions, and the simulations excluded from the study (non-significant SEMs) respectively. As well 


\section{COMPARISON OF MODEL FIT INDICES}

as the significance of the models included in the study, it was found that fit indices also have good fit and acceptable fit.

The comparative summarized table of model fit indices based on estimation techniques ( $p$-values) is given in Table 2. While no significant differentiation was identified in respect to $\chi^{2} / v$ ratio obtained from Model 1 based on the estimation techniques and RMSEA indices, differentiations were identified in SRMR, TLI and CFI. Although the CFI was the least affected one from the estimation techniques among the model fit indices which were identified to have differentiations, SRMR was the most affected one. No significant differentiation between the normal theory techniques MLE and GLS or between $\mathrm{SB}_{-} \chi^{2}$ and each normal theory was found in respect to CFI. However, CFI obtained with ADF technique was identified to be different from those achieved by the other techniques. In terms of TLI, no significant differentiation was determined between MLE and $\mathrm{SB}_{-} \chi^{2}$ techniques and, as for SRMR index, between MLE and GLS techniques (Table 2).

When the entirety of the model fit indices were examined based on the estimation techniques in the structure given in Model 2, it was found that $\chi^{2} / v$ ratio index was different compared to GLS and ADF techniques, yet these produced similar values in all of the remaining techniques. As for the RMSEA and CFI indices, these were identified to show no difference compared to MLE, GLS and $\mathrm{SB}_{-} \chi^{2}$ techniques, yet all of the values obtained with $\mathrm{ADF}$ were different from those obtained with the other techniques. In respect to TLI, only MLE and $\mathrm{SB}_{-} \chi^{2}$ did not show any significant difference in between (Table 2 ).

Table 2. The comparative summarized table of model fit indices based on estimation techniques ( $p$-values for Tukey's HSD)

\begin{tabular}{|c|c|c|c|c|c|c|c|c|}
\hline \multirow[b]{2}{*}{ Technique } & \multicolumn{3}{|l|}{$\begin{array}{c}\text { Model } 1 \\
\text { Fit Indices }\end{array}$} & \multicolumn{5}{|c|}{$\begin{array}{c}\text { Model } 2 \\
\text { Fit Indices }\end{array}$} \\
\hline & $\chi^{2} / v^{\boldsymbol{a}_{\mathcal{E}}} \boldsymbol{S} \boldsymbol{R} \boldsymbol{M} \boldsymbol{R}^{\alpha} \boldsymbol{R} \boldsymbol{M S E A ^ { \alpha \mathcal { E } }}$ & $T L P^{x}$ & $C F P^{a}$ & $\chi^{2} / v^{x}$ & SRMR & RMSEA $^{a}$ & $T L I^{x}$ & $C F^{\prime \prime}$ \\
\hline$M L E-G L S$ & 0.191 & $<0.001$ & 0.372 & 0.42 & $<0.001$ & 0.471 & $<0.001$ & 0.72 \\
\hline$M L E-A D F$ & $<0.001$ & $<0.001$ & $<0.001$ & 0.068 & $<0.001$ & 0.022 & $<0.001$ & $<0.001$ \\
\hline$M L E-S B-\chi^{2}$ & $<0.001$ & 1.000 & 0.999 & 1.000 & $<0.001$ & 0.999 & 1.000 & 0.999 \\
\hline$G L S-A D F$ & $<0.001$ & 0.002 & 0.038 & $<0.001$ & $<0.001$ & $<0.001$ & $<0.001$ & $<0.001$ \\
\hline$G L S-S B \_\chi^{2}$ & $<0.001$ & $<0.001$ & 0.457 & 0.401 & $<0.001$ & 0.551 & $<0.001$ & 0.629 \\
\hline$A D F-S B-\chi^{2}$ & $<0.001$ & $<0.001$ & $<0.001$ & 0.074 & $<0.001$ & 0.015 & $<0.001$ & $<0.001$ \\
\hline
\end{tabular}

MLE: Maximum Likelihood Estimation; GLS: Generalized Least Squares; ADF: Asymptotically Distribution Free; SB_ $X^{2}$ : Satorra-Bentler Scaled Chi-Square; $X^{2} / v$ :(Chi-Square test statistic/degree of freedom) ratio; SRMR: Standardized Root Mean Square Residual; RMSEA: Root Mean Square Error of Approximation; TLI: Tucker Lewis Index; CFI: Comparative Fit Index; ${ }^{2}$ : Ranked Value; Degree of Freedom of Model $1\left(v_{1}\right)=6$; Degree of

Freedom of Model $2\left(v_{2}\right)=14 ; £: p>0.05$ value for Factorial ANOVA 


\section{CANGUR \& ERCAN}

Table 3. The comparative summarized table of model fit indices based on sample sizes ( $p$-values for Tukey's HSD)

\begin{tabular}{|c|c|c|c|c|c|c|c|c|c|c|}
\hline \multirow[b]{2}{*}{$\begin{array}{c}\text { Sample } \\
\text { Size }\end{array}$} & \multicolumn{5}{|c|}{$\begin{array}{c}\text { Model } 1 \\
\text { Fit Indices } \\
\end{array}$} & \multicolumn{5}{|c|}{$\begin{array}{c}\text { Model } 2 \\
\text { Fit Indices } \\
\end{array}$} \\
\hline & $\chi^{2} / v^{\text {a }}$ & SRMR $^{\mathrm{a}}$ & RMSEA $^{\mathrm{a}}$ & TLI $^{\mathrm{x}}$ & $\mathrm{CFI}^{\mathrm{a}}$ & $\chi^{2} / v^{a}$ & $\mathrm{SRMR}^{\mathrm{a}}$ & RMSEA $^{a}$ & TLI $^{x}$ & $\mathrm{CFI}$ \\
\hline $100-500$ & 0.006 & $<0.001$ & 0.005 & $<0.001$ & $<0.001$ & 0.005 & $<0.001$ & 0.217 & $<0.001$ & 0.004 \\
\hline $100-1000$ & 0.001 & $<0.001$ & 0.049 & $<0.001$ & $<0.001$ & 0.024 & $<0.001$ & 0.003 & $<0.001$ & $<0.001$ \\
\hline $500-1000$ & 0.786 & $<0.001$ & 0.705 & $<0.001$ & 0.862 & 0.863 & $<0.001$ & 0.236 & $<0.001$ & 0.126 \\
\hline
\end{tabular}

$\left(x^{2} / v\right)$ : (Chi-Square test statistic/degree of freedom) ratio; SRMR: Standardized Root Mean Square Residual; RMSEA: Root Mean Square Error of Approximation; TLI: Tucker - Lewis Index; CFI: Comparative Fit Index; ${ }^{a}$ : Ranked Value; Degree of Freedom of Model $1\left(v_{1}\right)=6$; Degree of Freedom of Model $2\left(v_{2}\right)=14$

The summarized comparative table of model fit indices based on sample size ( $p$-values) is given in Table 3. The index values of SRMR and TLI obtained from Model 1 under multivariate normality condition was found to be significantly different according to sample sizes. However, while $\chi^{2} / v$ ratio, RMSEA and CFI obtained with a sample size of 100 units were observed to be significantly different from those obtained with the sample sizes of 500 and 1000 units, no significant differentiation was observed in none of the three indices obtained in sample sizes of 500 and 1000 units. With the increasing sample size, and in particular, when the sample size was above 500 units, it can be said that no significant change is seen in $\chi^{2} / v$, RMSEA and CFI values. All model fit indices showed significant differences based on sample size. However, while no significant differentiation was identified when they were examined in respect to $\chi^{2} / v$ ratio, RMSEA and CFI values based on large sample size $(n>500)$, significant differentiation was determined according to small and large sample sizes (100 and 1000). Additionally, it was found that there is no difference between the values obtained with small sample sizes (100 and 500) in RMSEA.

\section{Discussion}

The empirical evaluation of the proposed models is an important aspect of theory development process. It was determined that the $\chi^{2} / v$ ratio index based on the structures given in Model 1 and Model 2 was not affected from MLE and SB_ $\chi^{2}$ techniques, and RMSEA and CFI were not affected from MLE, GLS and SB_ $\chi^{2}$. TLI was determined to be insensitive to MLE and $\mathrm{SB}_{-} \chi^{2}$ techniques, yet SRMR index was affected from all estimation techniques. When the compliance of our findings with the literature is evaluated on the basis of models, it is seen that they 


\section{COMPARISON OF MODEL FIT INDICES}

are generally in compliance with the results of the studies conducted by Sugawara and MacCallum (1993), Hu and Bentler (1998, 1999), Fan et al. (1999), and Lei and Lomax (2005) yet entirely incompliant with the results produced by Ding et al. (1995).

When both model structures are taken into consideration in multivariate normal distribution condition and in the event of studying with large sample size; $\chi^{2} / v$ rate, RMSEA and CFI were determined to be independent from sample size while SRMR and TLI were dependent. When the compliance of our findings with the literature is examined on the basis of models, it was generally in parallel to the study results produced by Lacobucci (2010), Herzog et al. (2009), Jackson, (2001, 2007), Beauducel and Wittmann (2005), Curran et al. (2003), Kenny and McCoach (2003), Curran et al. (2002), Hu and Bentler (1999), Fan et al. (1999), Ding et al. (1995), Marsh and Balla (1994). Yet our findings except RMSEA were quite different from the study results of Fan and Sivo (2007). Furthermore, Rigdon (1996) emphasized the requirement to prefer RMSEA with large sample sizes and researches conducted to develop theory in his study in which RMSEA and CFI were compared.

The difference of model structure was accepted as an exogenous factor rather than a primary effect. Therefore, it can be stated that particular model fit indices obtained with only $\mathrm{ADF}$ technique are negatively affected from the increase of the number of latent variables when the result is evaluated in respect to the factors examined in this study.

In conclusion, it would be appropriate to prefer $\chi^{2} / v$ ratio, RMSEA and CFI in the event of studying with large samples and utilization of MLE, GLS and SB $\_\chi^{2}$ techniques under multivariate normal distribution condition. Furthermore, we do not recommend using SRMR in model fit research as it is the most affected index from estimation technique and sample size.

\section{References}

Beauducel, A., \& Wittmann, W. W. (2005). Simulation study on fit indices in confirmatory factor analysis based on data with slightly distorted simple structure. Structural Equation Modeling: A Multidisciplinary Journal, 12(1), 4175. doi:10.1207/s15328007sem1201_3

Bentler, P. M. (1994). A testing method for covariance structure analysis. In T. W. Anderson, K. T. Fang \& I. Olkin (Eds.), Multivariate Analysis and Its 


\section{CANGUR \& ERCAN}

Applications. Institute of Mathematical Statistics Lecture Notes-Monograph series, 24, 123-136. doi:10.1214//nms/1215463790

Bentler, P. M., \& Dudgeon, P. (1996). Covariance structure analysis: statistical practice, theory and directions. Annual Review of Psychology, 47(1), 563-592. doi:10.1146/annurev.psych.47.1.563

Boomsma, A., \& Hoogland, J. J. (2001). The robustness of LISREL modeling revisited. In R. Cudeck, S. Du Toit \& D. Sörbom (Eds.), Structural equation models: Present and future (pp. 139-168). Chicago: Scientific Software International Inc.

Chen, F. F. (2007). Sensitivity of goodness of fit indexes to lack of measurement invariance. Structural Equation Modeling: A Multidisciplinary Journal, 14(3), 464-504. doi:10.1080/10705510701301834

Chou, C. P., \& Bentler, P. M. (1995). Estimates and tests in structural equation modeling. In R. H. Hoyle (Ed.), Structural equation modeling: Concepts, issues, and applications (pp. 37-55). Thousand Oaks: Sage Publications.

Curran, P. J., Bollen, K. A., Paxton, P., Kirby, J., \& Chen, F. (2002). The noncentral chi-square distribution in misspecified structural equation models: Finite sample results from a Monte Carlo simulation. Multivariate Behavioral Research,37(1), 1-36. doi:10.1207/S15327906MBR3701_01

Curran, P. J., Bollen, K. A., Chen, F., Paxton, P., \& Kirby, J. (2003). Finite sampling properties of the point estimates and confidence intervals of the RMSEA. Sociological Methods \& Research, 32(2), 208-252.

doi:10.1177/0049124103256130

Ding, L., Velicer, W. F., \& Harlow, L. L. (1995). Effects of estimation methods, number of indicators per factor, and improper solutions on structural equation modeling fit indices. Structural Equation Modeling: A Multidisciplinary Journal, 2(2), 119-143. doi:10.1080/10705519509540000

Fan, X., Thompson, B., \& Wang, L. (1999). Effects of sample size, estimation methods, and model specification on structural equation modeling fit indexes. Structural Equation Modeling: A Multidisciplinary Journal, 6(1), 56-83. doi:10.1080/10705519509540000

Fan, X., \& Sivo, S. A. (2007). Sensitivity of fit indices to model misspecification and model types. Multivariate Behavioral Research, 42(3), 509529. doi:10.1080/00273170701382864 


\section{COMPARISON OF MODEL FIT INDICES}

Finney, S. J., \& Distefano, C. (2006). Nonnormal and categorical data in structural equation modeling. In G. R. Hancock \& R. O. Mueller (Eds.), Structural equation modelling: second course (pp. 269-314). Greenwich: Information Age Publishing.

Gerbing, D. W., \& Anderson, J. C. (1992). Monte Carlo evaluations of goodness of fit indices for structural equation models. Sociological Methods and Research, 21(2), 132-160. doi:10.1177/0049124192021002002

Herzog, W., \& Boomsma, A. (2009). Small-sample robust estimators of non-centrality-based and incremental model fit. Structural Equation Modeling: A Multidisciplinary Journal, 16(1), 1-27. doi:10.1080/10705510802561279

Hu, L. T., \& Bentler, P. M. (1998). Fit indices in covariance structure modeling: Sensitivity to under parameterized model misspecification. Psychological Methods, 3(4), 424-453. doi:10.1037/1082-989X.3.4.424

Hu, L. T., \& Bentler, P. M. (1999). Cutoff criteria for fit indexes in covariance structure analysis: Conventional criteria versus new alternatives. Structural Equation Modeling: A Multidisciplinary Journal, 6(1), 1-55. doi:10.1080/10705519909540118

Jackson, D. L. (2001). Sample size and number of parameter estimates in maximum likelihood confirmatory factor analysis: A Monte Carlo investigation. Structural Equation Modeling: A Multidisciplinary Journal, 8(2), 205-223. doi:10.1207/S15328007SEM0802_3

Jackson, D. L. (2007). The effect of the number of observations per parameter in misspecified confirmatory factor analytic models. Structural Equation Modeling: A Multidisciplinary Journal, 14(1), 48-76.

doi:10.1080/10705510709336736

Kenny, D. A., \& McCoach, D. B. (2003). Effect of the number of variables on measures of fit in structural equation modeling. Structural Equation Modeling: A Multidisciplinary Journal, 10(3), 333-351. doi:10.1207/S15328007SEM1003_1

Kline, R. B. (2011). Principles and practice of structural equation modeling. 3rd edition. New York: The Guilford Press.

Lacobucci, D. (2010). Structural equations modeling: Fit indices, sample size, and advanced topics. Journal of Consumer Psychology, 20(1), 90-98. doi:10.1016/j.jcps.2009.09.003

Lei, M., \& Lomax, R. G. (2005). The effect of varying degrees of nonnormality in structural equation modelling. Structural Equation Modeling: A Multidisciplinary Journal, 12(1), 1-27. doi:10.1207/s15328007sem1201_1 


\section{CANGUR \& ERCAN}

Lee, S. Y. (2007). Structural equation modeling: A bayesian approach. New York, NJ: John Wiley \& Sons.

Marsh, H. W., \& Balla, J. R. (1994). Goodness of fit indices in confirmatory factor analysis: The effects of sample size and model parsimony. Quality \& Quantity, 28(2), 185-217. doi:10.1007/BF01102761

Marsh, H. W., Hau, K. T., \& Wen, Z. (2004). In search of golden rules: Comment on hypothesis-testing approaches to setting cutoff values for fit indexes and dangers in overgeneralizing Hu and Bentler's (1999) findings. Structural Equation Modeling: A Multidisciplinary Journal, 11(3), 320-341. doi:10.1207/s15328007sem1103_2

Rigdon, E. E. (1996). CFI versus RMSEA: A comparison of two fit indexes for structural equation modeling. Structural Equation Modeling: A Multidisciplinary Journal, 3(4), 369-379. doi:10.1080/10705519609540052

Schermelleh-Engel, K., \& Moosbrugger, H. (2003). Evaluating the fit of structural equation models: tests of significance and descriptive goodness-of-fit measures. Methods of Psychological Research Online, 8(2), 23-74.

Sivo, S. A., Fan, X., Witta, E. L., \& John, T. (2006). The search for "optimal" cutoff properties: Fit index criteria in structural equation modeling. The Journal of Experimental Education, 74(3), 267-288. doi:10.3200/JEXE.74.3.267-288

Sugawara, H. M., \& MacCallum, R. C. (1993). Effect of estimation method on incremental fit indexes for covariance structure models. Applied Psychological Measurement, 17(4), 365-377. doi:10.1177/014662169301700405

Wang, L., Fan, X., \& Willson, V. L. (1996). Effects of nonnormal data on parameter estimates and fit indices for a model with latent and manifest variables: An empirical study. Structural Equation Modeling: A Multidisciplinary Journal, 3(3), 228-247. doi:10.1080/10705519609540042 